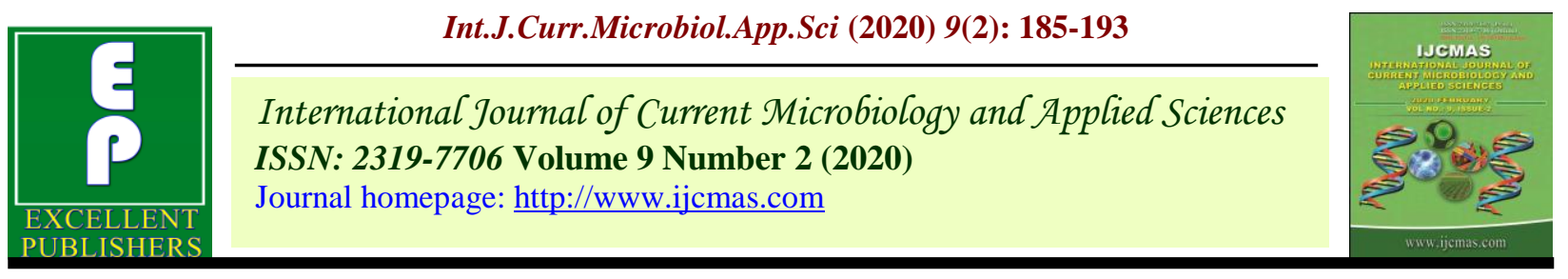

Original Research Article

https://doi.org/10.20546/ijcmas.2020.902.023

\title{
Survey Report: Performance Evaluation of Deenbandhu Biogas plants Installed in Various Regions of Punjab, India
}

\author{
Deepinder Singh Aulakh, Jasvarinder Chalotra* and S. S. Sooch \\ Department of Renewable Energy Engineering, COAE\&T, PAU, India \\ *Corresponding author
}

\begin{tabular}{|l|}
\hline Ke y w o r d s \\
Organic waste, \\
Survey \& \\
Inspection, \\
Deenbandhu biogas \\
model, Analysis \\
Report \\
\hline Article Info \\
\hline $\begin{array}{l}\text { Accepted: } \\
\text { 05 January } 2020 \\
\text { Available Online: } \\
\text { 10 February } 2020\end{array}$ \\
\hline
\end{tabular}

\section{A B S T R A C T}

Greenhouse gas emission is the biggest challenge to the society and responsible for major climatic changes these days. The non-renewable sources are responsible for this and should be replaced by the renewable once. Biogas is one of the most reliable and efficient renewable resource. Various organic wastes such as cattle dung, crop residues, human excreta, kitchen waste etc. are available in nature and can be easily used for biogas production. In this study, the survey was conducted by the engineers deputed by department of civil engineering, Punjab Agricultural University, Ludhiana, Punjab. The main objective of this survey was to evaluate the performance of family size biogas plant (Deenbandu model) already installed in the various regions of Punjab and to promote the use of biogas plant instead of LPG cylinders. 100 plants were randomly selected from various regions and on the basis of user experience and data collected a report was prepared.

\section{Introduction}

Energy is a vital input for economic growth in agricultural and industrial field. The depletion of fossil fuels (non-renewable energy)is at alarming rate and the cost of environment protection is also increasing. Renewable resources technology is of utmost importance for balance and healthier environment for living. The renewable resources such as sun, wind, biomass, geothermal and tidal energy are abundant resources of energy provided by nature. Biomass refers to the solid carbonaceous material derived from plants and animals. Biomass does not add $\mathrm{CO}_{2}$ to the atmosphere as it absorbs the same amount of carbon in growing plants as it releases when consumed as fuel. Biomass fuel is used maximum in rural households and little bit in urban dwellings ${ }^{1}$.

Biogas from biomass is one of the most sustainable and effective source of energy which endures for a long time. Biogas is 
derived by anaerobic digestion of organic wastes such as cattle dung, crop residues, human excreta, kitchen waste etc ${ }^{1}$. Anaerobic digestion not only provides valuable fuel but also enhances the fertilizer value of residue. It also provides environment friendly, safe, conventional and economical disposal method of waste. This process gives better results as compared to other waste treatment processes. Developing the technology for biogas production is subjected to well proven technique and economical aspects of the country. Biogas has both social and industrial advantages as it is safe fuel for cooking, lighting and engine running. The left over digested slurry is enriched manure, which can be used in agricultural land. Biogas consists of methane $\left(\mathrm{CH}_{4}\right)$, carbon dioxide $\left(\mathrm{CO}_{2}\right)$ and traces of other gases such as $\mathrm{H}_{2}, \mathrm{CO}, \mathrm{N}_{2}$ and $\mathrm{H}_{2} \mathrm{~S}^{2}$. Methane is the main component of biogas which is used for purposes as it has same thermal efficiency compared to LPG (butane gas). Table 1 shows the thermal efficiency of various fuels with calorific values $^{2}$. For the production of biogas, different models are developed such as KVIC, Janta and Deenbandu model. Out of these, Deenbandhu modelis the most economical and smallest family size plant.

Aim of the study was to conduct the survey over selected installed biogas plants in the region of Punjab so as to know the working condition, problem faced and awareness among the people.

\section{Materials and Methods}

Survey Performa:- Initially, the perform a including various points such as name and address of beneficiary, no. of cattle owned, capacity, installation year, operational and structural problems of the plant for data collection was prepared.

Location selection:-The selection of location, installation and provision of after service is done with the help of the masons and selfemployed workers (SEWs) trained by the department. Based upon the information provided by the masons and SEWs, survey was conducted. In this process, engineers visit the selected spot and conduct the survey in contact mode with every individual person holding plant.

Deenbandhu biogas plant design: Figure 1 represents Deenabndhu biogas plant design model $^{2}$. This is the most popular and successful type of family size plant the in the region of Punjab. It is very economical and requires least space among the other designs. The major factors affecting the production of biogas are the temperature and $\mathrm{pH}$. The temperature is required to be in the range of $35^{\circ} \mathrm{C}-45^{\circ} \mathrm{C}$ but unfortunately, the process retards below $10^{\circ} \mathrm{C}$. On the other hand, $\mathrm{pH}$ factor should be in between 6.5 to 7.5 inside the digester ${ }^{3}$. For biogas generation, daily $25 \mathrm{~kg}$ per $\mathrm{m}^{3}$ of cow dung is required. The cow dung and water is mixed in the ratio $1: 1$ and added to the digester. These plants are installed in various sizes $1 \mathrm{~m}^{3}, 2 \mathrm{~m}^{3}, 3 \mathrm{~m}^{3}, 4 \mathrm{~m}^{3}$ and $6 \mathrm{~m}^{3}$ depending upon the number of members and animals present in the house. Mostly, $4 \mathrm{~m}^{3}$ and $6 \mathrm{~m}^{3}$ are more popular size of plants which are sufficient for 8-12 members of the family ${ }^{2}$. The government also provides subsidy on these sizes of plants.

Analysis:- After collection of data provided by the owner of plant from various regions. Excel sheet was prepared for the analysis.

\section{Results and Discussion}

Survey over 100 installed biogas plants are conducted and reported in the table 2 .

It has been observed from the survey that most of the people prefer $6 \mathrm{~m}^{3}$ of biogas plant over other sizes due to high number of family members and secondly, keeping in mind consistent progression in the family members. 
Another noticeable factor from the survey was that with the advancement in the time and a building sense of awareness among the people there has been a tremendous increase in the installation of biogas plant. The yearly growth of biogas plants installed is shown in Fig. 2.

Table.1 Comparison of calorific value and thermal efficiency of various fuels are

\begin{tabular}{|c|l|c|c|}
\hline $\begin{array}{c}\text { Sr. } \\
\text { No. }\end{array}$ & \multicolumn{1}{|c|}{ Name of fuel } & $\begin{array}{c}\text { Thermal Efficiency } \\
\text { (KCal) }\end{array}$ & Calorific value (KCal) \\
\hline $\mathbf{1}$ & Biogas $\left(\mathrm{m}^{3}\right)$ & 60 & 4713 \\
\hline $\mathbf{2}$ & Fire Wood $(\mathrm{kg})$ & 17.3 & 4708 \\
\hline $\mathbf{3}$ & Cow dung cake $(\mathrm{kg})$ & 17.3 & 2092 \\
\hline $\mathbf{4}$ & LPG(Butane gas) $(\mathrm{kg})$ & 60 & 10662 \\
\hline $\mathbf{5}$ & Charcoal $(\mathrm{kg})$ & 28 & 6930 \\
\hline
\end{tabular}

Table.2 Survey data of biogas plants

\begin{tabular}{|c|c|c|c|c|c|c|c|c|}
\hline \multirow{3}{*}{$\begin{array}{l}\text { Sr. } \\
\text { No. }\end{array}$} & \multirow{3}{*}{$\begin{array}{c}\text { Name \& } \\
\text { Village }\end{array}$} & \multirow[t]{3}{*}{ District } & \multirow{3}{*}{$\begin{array}{l}\text { No. of } \\
\text { Cattle } \\
\text { owned }\end{array}$} & \multirow{3}{*}{$\begin{array}{c}\text { Capacity } \\
\text { of plant } \\
\left(\mathbf{m}^{3}\right)\end{array}$} & \multirow{3}{*}{$\begin{array}{c}\text { Year of } \\
\text { installation }\end{array}$} & \multicolumn{3}{|c|}{ Status of plant } \\
\hline & & & & & & \multirow{2}{*}{$\begin{array}{c}\text { Working } \\
\text { satisfactorily } \\
\text { / Partially }\end{array}$} & \multicolumn{2}{|c|}{ Not working due to } \\
\hline & & & & & & & $\begin{array}{c}\text { Structural } \\
\text { problem }\end{array}$ & $\begin{array}{c}\text { Operational } \\
\text { problem }\end{array}$ \\
\hline 1. & $\begin{array}{l}\text { Lekh Singh } \\
\text { Vill:Charik }\end{array}$ & Moga & 7 & 6 & 2015 & Satisfactorily & -- & -- \\
\hline 2. & $\begin{array}{c}\text { Gurbachan Singh } \\
\text { Vill:Charik }\end{array}$ & Moga & 8 & 6 & 2015 & Satisfactorily & -- & -- \\
\hline 3. & $\begin{array}{c}\text { Gurnek Singh } \\
\text { Vill:Charik }\end{array}$ & Moga & 4 & 6 & 2014 & Satisfactorily & -- & -- \\
\hline 4. & $\begin{array}{c}\text { Hardev Singh } \\
\text { Vill:Charik }\end{array}$ & Moga & 15 & 6 & 2015 & Satisfactorily & -- & -- \\
\hline 5. & $\begin{array}{l}\text { Baljinder Singh } \\
\text { Vill:Charik }\end{array}$ & Moga & 6 & 6 & 2016 & Satisfactorily & -- & -- \\
\hline 6. & $\begin{array}{l}\text { Sarabjit Singh } \\
\text { Vill:khai }\end{array}$ & Moga & 5 & 6 & 2013 & Satisfactorily & -- & -- \\
\hline 7. & $\begin{array}{c}\text { Jagdev Singh } \\
\text { Vill:Khai }\end{array}$ & Moga & 6 & 6 & 2014 & Satisfactorily & -- & -- \\
\hline 8. & $\begin{array}{c}\text { Karam Singh } \\
\text { Vill:Khai }\end{array}$ & Moga & 10 & 6 & 2016 & Satisfactorily & -- & -- \\
\hline 9. & $\begin{array}{c}\text { Kirandeep Kaur } \\
\text { Vill:Kha }\end{array}$ & Moga & 4 & 6 & 2016 & Satisfactorily & -- & -- \\
\hline 10. & $\begin{array}{c}\text { Baljit Kaur } \\
\text { Vill: Khai }\end{array}$ & Moga & 10 & 6 & 2015 & Satisfactorily & -- & -- \\
\hline 11. & $\begin{array}{l}\text { Avtar Singh } \\
\text { Vill:Daburji }\end{array}$ & Moga & 5 & 6 & 2015 & Satisfactorily & -- & -- \\
\hline 12. & $\begin{array}{c}\text { Suksar Singh } \\
\text { Vill:Daburji }\end{array}$ & Moga & 7 & 6 & 2009 & Satisfactorily & -- & -- \\
\hline
\end{tabular}




\begin{tabular}{|c|c|c|c|c|c|c|c|c|}
\hline 13. & $\begin{array}{l}\text { Pehal Singh } \\
\text { Vill:Daburji }\end{array}$ & Moga & 8 & 6 & 2011 & Satisfactorily & -- & -- \\
\hline 14. & $\begin{array}{l}\text { Labh Singh } \\
\text { Vill:Daburji }\end{array}$ & Moga & 6 & 6 & 2012 & Satisfactorily & -- & -- \\
\hline 15. & $\begin{array}{c}\text { Jagtar Singh } \\
\text { Vill:Korewala }\end{array}$ & Moga & 7 & 6 & 2016 & Satisfactorily & -- & -- \\
\hline 16. & $\begin{array}{c}\text { Malkit Singh } \\
\text { Vill:Korewala }\end{array}$ & Moga & 6 & 6 & 2011 & Satisfactorily & -- & -- \\
\hline 17. & $\begin{array}{l}\text { Harnam Singh } \\
\text { Vill:Korewala }\end{array}$ & Moga & 8 & 6 & 2008 & Satisfactorily & -- & -- \\
\hline 18. & $\begin{array}{l}\text { Nirmal Singh } \\
\text { Vill:Korewala }\end{array}$ & Moga & 40 & 6 & 2008 & Satisfactorily & -- & -- \\
\hline 19. & $\begin{array}{l}\text { Paramjit Singh } \\
\text { Vill:Korewala }\end{array}$ & Moga & 10 & 6 & 2007 & Satisfactorily & -- & -- \\
\hline 20. & $\begin{array}{l}\text { Kulwant Singh } \\
\text { Vill:Korewala }\end{array}$ & Moga & 9 & 6 & 2008 & Satisfactorily & -- & -- \\
\hline 21. & $\begin{array}{l}\text { Sukha Singh } \\
\text { Vill:Korewala }\end{array}$ & Moga & 4 & 6 & 2007 & Satisfactorily & -- & -- \\
\hline 22. & $\begin{array}{l}\text { Darshan Singh } \\
\text { Vill: Kaulgarh }\end{array}$ & $\begin{array}{l}\text { Fatehgarh } \\
\text { Sahib }\end{array}$ & $18-20$ & 6 & 1996 & Satisfactorily & -- & -- \\
\hline 23. & $\begin{array}{l}\text { Nichatar Singh } \\
\text { Vill:Kaulgarh }\end{array}$ & $\begin{array}{l}\text { Fatehgarh } \\
\text { Sahib }\end{array}$ & 4 & 6 & 1991 & Satisfactorily & -- & -- \\
\hline 24. & $\begin{array}{l}\text { Hardeep Singh } \\
\text { Vill:Kaulgarh }\end{array}$ & $\begin{array}{l}\text { Fatehgarh } \\
\text { Sahib }\end{array}$ & $10-12$ & 8 & 1985 & Satisfactorily & -- & -- \\
\hline 25. & $\begin{array}{l}\text { Jatinder Singh } \\
\text { Vill:Kaulgarh }\end{array}$ & $\begin{array}{l}\text { Fatehgarh } \\
\text { Sahib }\end{array}$ & $10-11$ & 6 & 1990 & Satisfactorily & -- & -- \\
\hline 26. & $\begin{array}{l}\text { Baljeet Singh } \\
\text { Vill:Kaulgarh }\end{array}$ & $\begin{array}{l}\text { Fatehgarh } \\
\text { Sahib }\end{array}$ & 4 & 6 & 2009 & Satisfactorily & -- & -- \\
\hline 27. & $\begin{array}{l}\text { Saudagar Singh } \\
\text { Vill:Kaulgarh }\end{array}$ & $\begin{array}{l}\text { Fatehgarh } \\
\text { Sahib }\end{array}$ & 12 & 6 & 1995 & Satisfactorily & -- & -- \\
\hline 28. & $\begin{array}{l}\text { Achhra Singh } \\
\text { Vill:Kaulgarh }\end{array}$ & $\begin{array}{l}\text { Fatehgarh } \\
\text { Sahib }\end{array}$ & $15-16$ & 6 & 1993 & Satisfactorily & -- & -- \\
\hline 29. & $\begin{array}{l}\text { Amolak Singh } \\
\text { Vill:Kaulgarh }\end{array}$ & $\begin{array}{l}\text { Fatehgarh } \\
\text { Sahib }\end{array}$ & $10-12$ & 6 & 1994 & Satisfactorily & -- & -- \\
\hline 30. & $\begin{array}{l}\text { Karmolak Singh } \\
\text { Vill:Kaulgarh }\end{array}$ & $\begin{array}{l}\text { Fatehgarh } \\
\text { Sahib }\end{array}$ & $20-22$ & 6 & 1994 & Satisfactorily & -- & -- \\
\hline 31. & $\begin{array}{l}\text { Sandeep Singh } \\
\text { Vill: Kaulgarh }\end{array}$ & $\begin{array}{l}\text { Fatehgarh } \\
\text { Sahib }\end{array}$ & 7 & 6 & 1991 & Uncommisioned & -- & -- \\
\hline 32. & $\begin{array}{l}\text { Amrik Singh } \\
\text { Vill:Kaulgarh }\end{array}$ & $\begin{array}{l}\text { Fatehgarh } \\
\text { Sahib }\end{array}$ & $11-12$ & 6 & 1992 & Satisfactorily & -- & -- \\
\hline 33. & $\begin{array}{l}\text { Baljinder Singh } \\
\text { Vill:Kaulgarh }\end{array}$ & $\begin{array}{l}\text { Fatehgarh } \\
\text { Sahib }\end{array}$ & $3-4$ & 6 & 2012 & Satisfactorily & -- & -- \\
\hline 34. & $\begin{array}{l}\text { Kamaljeet Singh } \\
\text { Vill:Kaulgarh }\end{array}$ & $\begin{array}{l}\text { Fatehgarh } \\
\text { Sahib }\end{array}$ & 15 & 6 & 2005 & Satisfactorily & -- & -- \\
\hline 35. & $\begin{array}{l}\text { Sant Ram } \\
\text { Vill:Ajroar }\end{array}$ & Patiala & $10-11$ & 6 & 2009 & Satisfactorily & -- & -- \\
\hline 36. & $\begin{array}{l}\text { Gurcharan Singh } \\
\text { Vill: Ajroar }\end{array}$ & Patiala & $5-7$ & 6 & 2012 & Satisfactorily & -- & -- \\
\hline 37. & $\begin{array}{c}\text { Kartar Singh } \\
\text { Vill: Nathu } \\
\text { Manjra }\end{array}$ & Patiala & 5 & 6 & 2012 & Satisfactorily & -- & -- \\
\hline 38. & $\begin{array}{c}\text { Jarnail Singh Vill: } \\
\text { Ajroar }\end{array}$ & Patiala & 8 & 6 & 2009 & Partially & -- & Under Feeding \\
\hline
\end{tabular}




\begin{tabular}{|c|c|c|c|c|c|c|c|c|}
\hline 39. & $\begin{array}{c}\text { Gurtej Singh Vill: } \\
\text { Ajrawar }\end{array}$ & Patiala & 4 & 6 & 2012 & Satisfactorily & -- & -- \\
\hline 40. & $\begin{array}{c}\text { Tara Singh Vill: } \\
\text { Mandli }\end{array}$ & Patiala & 3 & 6 & 2011 & Satisfactorily & -- & -- \\
\hline 41. & $\begin{array}{l}\text { Harbans Singh } \\
\text { Vill: Mandli }\end{array}$ & Patiala & 4 & 6 & 2011 & Satisfactorily & -- & -- \\
\hline 42. & $\begin{array}{l}\text { Gurmukh Singh } \\
\text { Vill: Mehma }\end{array}$ & Patiala & 20 & 6 & 2014 & Satisfactorily & -- & -- \\
\hline 43. & $\begin{array}{c}\text { Jaswant Singh } \\
\text { Vill: } \\
\text { KehriGandiya }\end{array}$ & Patiala & 4 & 6 & 2013 & Satisfactorily & -- & -- \\
\hline 44. & $\begin{array}{c}\text { Balvir Singh Vill: } \\
\text { KehriGandiya }\end{array}$ & Patiala & $5-7$ & 6 & 2013 & Satisfactorily & -- & -- \\
\hline 45. & $\begin{array}{l}\text { Surinder Singh } \\
\text { Vill: Mehma }\end{array}$ & Patiala & $7-8$ & 6 & 2014 & Satisfactorily & -- & -- \\
\hline 46. & $\begin{array}{l}\text { Pritpal SinghVill: } \\
\text { KehriGandiya }\end{array}$ & Patiala & 10 & 6 & 2013 & Satisfactorily & -- & -- \\
\hline 47. & $\begin{array}{l}\text { Des Raj Vill: } \\
\text { Lochma }\end{array}$ & Patiala & $9-10$ & 6 & 2015 & Satisfactorily & -- & -- \\
\hline 48. & $\begin{array}{c}\text { Sawarn Singh } \\
\text { Vill: KherpurJatta }\end{array}$ & Patiala & 35 & 6 & 2013 & Satisfactorily & -- & -- \\
\hline 49. & $\begin{array}{c}\text { Shiv Ram } \\
\text { Vill:KherpurJatta }\end{array}$ & Patiala & $6-7$ & 6 & 2009 & Satisfactorily & -- & -- \\
\hline 50. & $\begin{array}{l}\text { Jawar Singh } \\
\text { Vill:Mehma }\end{array}$ & Patiala & $15-16$ & 6 & 2011 & Satisfactorily & -- & -- \\
\hline 51. & $\begin{array}{c}\text { JasdevSinghVill: } \\
\text { Saunti }\end{array}$ & $\begin{array}{l}\text { Fatehgarh } \\
\text { Sahib }\end{array}$ & $3-5$ & 6 & 2012 & Satisfactorily & -- & -- \\
\hline 52. & $\begin{array}{l}\text { Gurcharan Singh } \\
\text { Vill: Saunti }\end{array}$ & $\begin{array}{l}\text { Fatehgarh } \\
\text { Sahib }\end{array}$ & $2-4$ & 4 & 2011 & Satisfactorily & -- & -- \\
\hline 53. & $\begin{array}{l}\text { Rashvinder Singh } \\
\text { Vill: Saunti }\end{array}$ & $\begin{array}{l}\text { Fatehgarh } \\
\text { Sahib }\end{array}$ & $10-11$ & 6 & 2000 & Satisfactorily & -- & -- \\
\hline 54. & $\begin{array}{c}\text { Nibah Singh Vill: } \\
\text { Saunti }\end{array}$ & $\begin{array}{l}\text { Fatehgarh } \\
\text { Sahib }\end{array}$ & 6 & 6 & 2008 & Satisfactorily & -- & -- \\
\hline 55. & $\begin{array}{l}\text { Jasvinder Singh } \\
\text { Vill: Saunti }\end{array}$ & $\begin{array}{l}\text { Fatehgarh } \\
\text { Sahib }\end{array}$ & $2-4$ & 6 & 2015 & Satisfactorily & -- & -- \\
\hline 56. & $\begin{array}{l}\text { Harvinder Singh } \\
\text { Vill: } \\
\text { MajriKishniwala }\end{array}$ & $\begin{array}{l}\text { Fatehgarh } \\
\text { Sahib }\end{array}$ & $6-7$ & 6 & 1998 & Partially & -- & Under Feeding \\
\hline 57. & $\begin{array}{c}\text { Hardam Singh } \\
\text { Vill: } \\
\text { MajriKishniwala }\end{array}$ & $\begin{array}{l}\text { Fatehgarh } \\
\text { Sahib }\end{array}$ & 4 & 6 & 1998 & Partially & -- & Under Feeding \\
\hline 58. & $\begin{array}{l}\text { Harvinder Singh } \\
\text { Vill: } \\
\text { MajriKishniwala }\end{array}$ & $\begin{array}{l}\text { Fatehgarh } \\
\text { Sahib }\end{array}$ & $10-11$ & 6 & 2004 & Satisfactorily & -- & -- \\
\hline 59. & $\begin{array}{l}\text { Jagtar Singh Vill: } \\
\text { MajriKishniwala }\end{array}$ & $\begin{array}{l}\text { Fatehgarh } \\
\text { Sahib }\end{array}$ & $20-22$ & 6 & 2014 & Satisfactorily & -- & -- \\
\hline 60. & $\begin{array}{l}\text { Avtar Singh } \\
\text { Vill: Pola }\end{array}$ & $\begin{array}{l}\text { Fatehgarh } \\
\text { Sahib }\end{array}$ & 5 & 4 & 2007 & Satisfactorily & -- & -- \\
\hline
\end{tabular}




\begin{tabular}{|c|c|c|c|c|c|c|c|c|}
\hline 61. & $\begin{array}{l}\text { Major Singh Vill: } \\
\text { PunjoliKhurd }\end{array}$ & $\begin{array}{l}\text { Fatehgarh } \\
\text { Sahib }\end{array}$ & 15 & 20 & 1997 & Satisfactorily & -- & -- \\
\hline 62. & $\begin{array}{c}\text { Jasvant Singh } \\
\text { Vill: } \\
\text { PunjoliKhurd }\end{array}$ & $\begin{array}{l}\text { Fatehgarh } \\
\text { Sahib }\end{array}$ & 11 & 10 & 2011 & Satisfactorily & -- & -- \\
\hline 63. & $\begin{array}{c}\text { Gurmeet Singh } \\
\text { Vill: } \\
\text { PunjoliKhurd }\end{array}$ & $\begin{array}{l}\text { Fatehgarh } \\
\text { Sahib }\end{array}$ & 6 & 4 & 2011 & Partially & -- & Under Feeding \\
\hline 64. & $\begin{array}{c}\text { Joginder Singh } \\
\text { Vill: } \\
\text { PunjoliKhurd }\end{array}$ & $\begin{array}{l}\text { Fatehgarh } \\
\text { Sahib }\end{array}$ & 5 & 4 & 2011 & Partially & Doom Crack & -- \\
\hline 65. & $\begin{array}{l}\text { Kulwant Singh } \\
\text { Vill: Panjoli } \\
\text { Kalan }\end{array}$ & $\begin{array}{l}\text { Fatehgarh } \\
\text { Sahib }\end{array}$ & 10 & 4 & 1998 & Satisfactorily & -- & -- \\
\hline 66. & $\begin{array}{l}\text { Jarnail Singh } \\
\text { Vill:Diala }\end{array}$ & Ludhiana & 7 & 6 & 2010 & Satisfactorily & -- & -- \\
\hline 67. & $\begin{array}{l}\text { Harpreet Singh } \\
\text { Vill:Diwala }\end{array}$ & Ludhiana & 8 & 6 & 2007 & Satisfactorily & -- & -- \\
\hline 68. & $\begin{array}{l}\text { Narinder Singh } \\
\text { Vill:Diwala }\end{array}$ & Ludhiana & 4 & 6 & 2014 & Satisfactorily & -- & -- \\
\hline 69. & $\begin{array}{l}\text { Kashmir Singh } \\
\text { Vill:Diwala }\end{array}$ & Ludhiana & 15 & 6 & 2012201 & Satisfactorily & -- & -- \\
\hline 70. & $\begin{array}{l}\text { Kuldeep Singh } \\
\text { Vill:Gharkhana }\end{array}$ & Ludhiana & 8 & 6 & 2006 & Satisfactorily & -- & -- \\
\hline 71. & $\begin{array}{l}\text { Santokh Singh } \\
\text { Vill:Gharkhana }\end{array}$ & Ludhiana & 2 & 6 & 1999 & Satisfactorily & -- & -- \\
\hline 72. & $\begin{array}{l}\text { SukhwinderSingh } \\
\text { Vill:Gharkhana }\end{array}$ & Ludhiana & 15 & 6 & 2011 & Satisfactorily & -- & -- \\
\hline 73. & $\begin{array}{c}\text { Jaspal Singh } \\
\text { Vill:Gharkhana }\end{array}$ & Ludhiana & 5 & 6 & 2015 & Satisfactorily & -- & -- \\
\hline 74. & $\begin{array}{l}\text { Harwinder Singh } \\
\text { Vill:Gharkhana }\end{array}$ & Ludhiana & 11 & 6 & 2014 & Satisfactorily & -- & -- \\
\hline 75. & $\begin{array}{c}\text { Teja Singh } \\
\text { Vill:Gharkhana }\end{array}$ & Ludhiana & 11 & 6 & 1996 & Satisfactorily & -- & -- \\
\hline 76. & $\begin{array}{l}\text { Khushwinder } \\
\text { Singh } \\
\text { Vill:Gharkhana }\end{array}$ & Ludhiana & 10 & 6 & 2000 & Satisfactorily & -- & -- \\
\hline 77. & $\begin{array}{l}\text { Gurdeep Singh } \\
\text { Vill:Gharkhana }\end{array}$ & Ludhiana & 4 & 6 & 2016 & Satisfactorily & -- & -- \\
\hline 78. & $\begin{array}{l}\text { Gursewak Singh } \\
\text { Vill:Gharkhana }\end{array}$ & Ludhiana & 10 & 6 & 2000 & Satisfactorily & -- & -- \\
\hline 79. & $\begin{array}{l}\text { Ujagar Singh } \\
\text { Vill:Gharkhana }\end{array}$ & Ludhiana & 8 & 6 & 1996 & Satisfactorily & -- & -- \\
\hline 80. & $\begin{array}{l}\text { Chand Singh } \\
\text { Vill:Gharkhana }\end{array}$ & Ludhiana & 6 & 6 & 2000 & Satisfactorily & -- & -- \\
\hline 81. & $\begin{array}{l}\text { Kesar Singh } \\
\text { Vill:Gosalan }\end{array}$ & Ludhiana & 8 & 6 & 1997 & Satisfactorily & -- & -- \\
\hline 82. & $\begin{array}{l}\text { Darshan Singh } \\
\text { Vill:Gosalan }\end{array}$ & Ludhiana & 10 & 6 & 2012 & Satisfactorily & -- & -- \\
\hline
\end{tabular}




\begin{tabular}{|c|c|c|c|c|c|c|c|c|}
\hline 83. & $\begin{array}{c}\text { Dev Singh } \\
\text { Vill:Gosalan }\end{array}$ & Ludhiana & 6 & 6 & 1992 & Satisfactorily & -- & -- \\
\hline 84. & $\begin{array}{l}\text { Swaran Singh } \\
\text { Vill:Gosalan }\end{array}$ & Ludhiana & 7 & 6 & 2009 & Satisfactorily & -- & -- \\
\hline 85. & $\begin{array}{l}\text { Mnapreet Singh } \\
\text { Vill:Gosalan }\end{array}$ & Ludhiana & 6 & 6 & 2011 & Satisfactorily & -- & -- \\
\hline 86. & $\begin{array}{l}\text { Balwinder Singh } \\
\text { Vill:Gosalan }\end{array}$ & Ludhiana & 8 & 6 & 2010 & Satisfactorily & -- & -- \\
\hline 87. & $\begin{array}{c}\text { Manjeet Singh } \\
\text { Vill:Bhagwanpura }\end{array}$ & Ludhiana & 30 & 6 & 2011 & Satisfactorily & -- & -- \\
\hline 88. & $\begin{array}{c}\text { Malkeet Singh } \\
\text { Vill:Bhagwanpura }\end{array}$ & Ludhiana & 10 & 6 & 2006 & Satisfactorily & -- & -- \\
\hline 89. & $\begin{array}{c}\text { Gulzar Singh } \\
\text { Vill:Bhagwanpura }\end{array}$ & Ludhiana & 20 & 6 & 2009 & Satisfactorily & -- & -- \\
\hline 90. & $\begin{array}{l}\text { Vikramjit Singh } \\
\text { Vill:Dalla }\end{array}$ & Jalandhar & 2 & 6 & 2013 & Satisfactorily & -- & -- \\
\hline 91. & $\begin{array}{l}\text { Kuldeep Singh } \\
\text { Vill:Dalla }\end{array}$ & Jalandhar & 6 & 6 & 2008 & Satisfactorily & -- & -- \\
\hline 92. & $\begin{array}{l}\text { Makhan Singh } \\
\text { Vill:Dalla }\end{array}$ & Jalandhar & 5 & 6 & 2001 & Satisfactorily & -- & -- \\
\hline 93. & $\begin{array}{l}\text { Harbhajan Singh } \\
\text { Vill:Kaimwala }\end{array}$ & Jalandhar & 4 & 6 & 2012 & Satisfactorily & -- & -- \\
\hline 94. & $\begin{array}{c}\text { Soocha Singh } \\
\text { Vill:Kaimwala }\end{array}$ & Jalandhar & 12 & 6 & 2000 & Satisfactorily & -- & -- \\
\hline 95. & $\begin{array}{c}\text { BalkarSingh } \\
\text { Vill:Kaimwala }\end{array}$ & Jalandhar & 8 & 6 & 2008 & Satisfactorily & -- & -- \\
\hline 96. & $\begin{array}{l}\text { Balwinder Singh } \\
\text { Vill:Kaimwala }\end{array}$ & Jalandhar & 2 & 6 & 2013 & Satisfactorily & -- & -- \\
\hline 97. & $\begin{array}{c}\text { Jasbir Singh } \\
\text { Vill:Kaimwala }\end{array}$ & Jalandhar & 2 & 6 & 2013 & Satisfactorily & -- & -- \\
\hline 98. & $\begin{array}{l}\text { Samund Singh } \\
\text { Vill:Kaimwala }\end{array}$ & Jalandhar & 6 & 6 & 2003 & Partially & -- & Under Feeding \\
\hline 99. & $\begin{array}{c}\text { Kulbiir Singh } \\
\text { Vill:Kaimwala }\end{array}$ & Jalandhar & 8 & 6 & 2012 & Satisfactorily & -- & -- \\
\hline 100. & $\begin{array}{l}\text { Darshan Singh } \\
\text { Vill:Mehsampur }\end{array}$ & Jalandhar & 3 & 6 & 1990 & Satisfactorily & -- & -- \\
\hline
\end{tabular}

Fig.1 Deenbandhu Biogas plant design

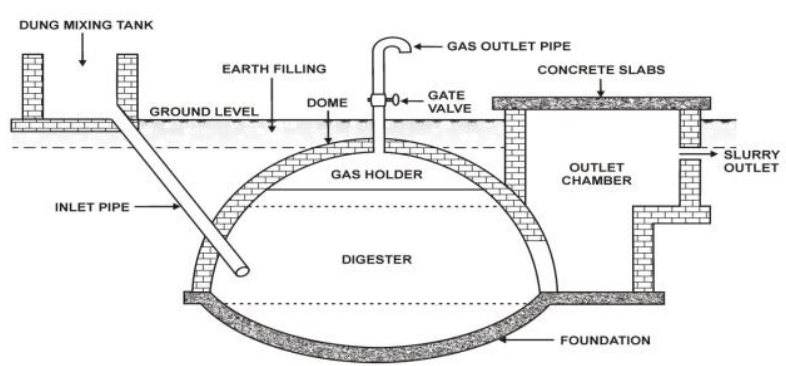


Fig.2 No. of biogas plant installed every year

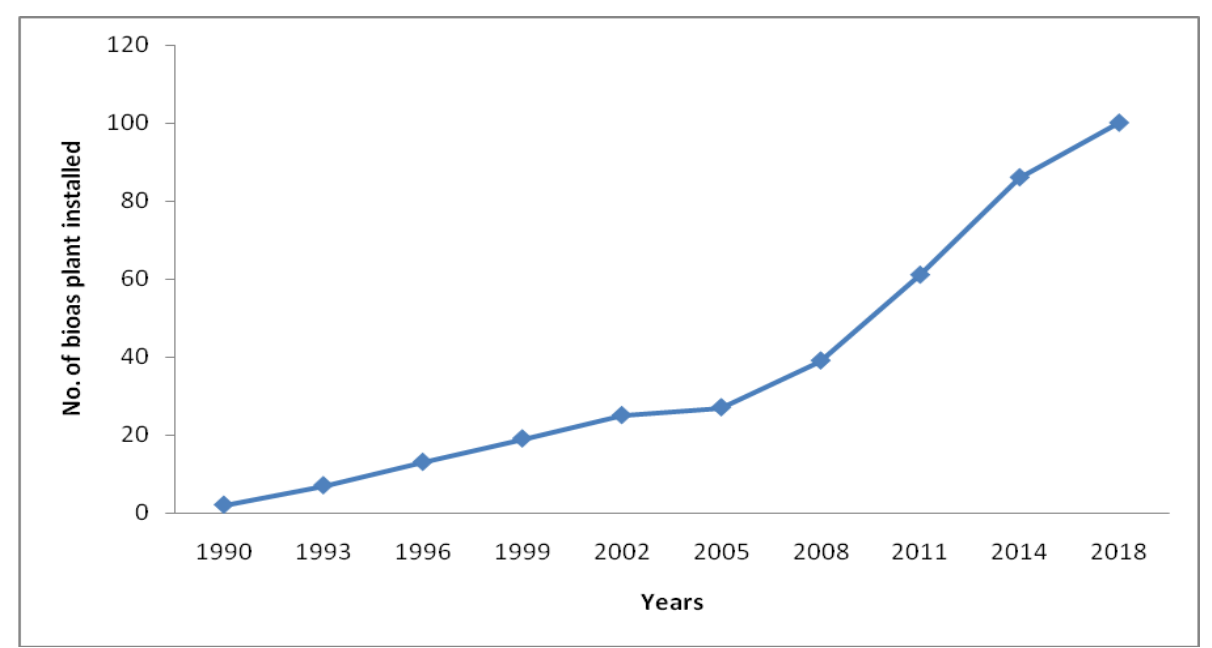

In order to overcome the problems posed by increasing energy demands and diminishing resources, it is necessary to switch to new alternate energy sources (environment friendly), so that the future generations can be prevented from facing various environment related issues like global warming, pollution, health hazards etc. Biogas has emerged out to be one of the most convenient and reliable energy resources. On the bases of survey, Deenbandhu biogas plants model is best for family and industry purposes as it can made upto any required size to meet the need.

\section{Future scope}

This is the largest alternative of LPG cylinder.

These models consume the harmful gases produces and convert into methane gas which is useful for cooking.

By increasing the size, the capacity of biogas generation increases and can be used for lighting lamps and power generation.

\section{Acknowledgement}

Authors would like to express their deepest gratitude to all the staff members and colleagues for their consistent support during the survey. One of the authors, Deepinder Singh would like to thank Dr. Mandeep Kaur (Asst. Professor, Baba Banda Singh Bahadur Engineering College, Fatehgarh Sahib) for her valuable suggestions during the work.

\section{References}

1. Kothari D P, Singal K C and Rajan R (2009) Renewable energy sources and emerging technologies, New Delhi, PHI Learning Pvt. Ltd..

2. Sooch S S (2018) important information on biogas technology, Ludhina, PAU.

3. Sooch S S and Gautam A (2013) Present status of renewable energy sources in Punjab. International Journal of Agriculture, Environment \& Technology 6(2): 317-33.

4. S S Sooch and Bhati J S (2016) Biogas plants for cooking and power generation. Dairy India 7: 263-66. 
How to cite this article:

Deepinder Singh Aulakh, JasvarinderChalotra and Sooch, S. S. 2020. Survey Report: Performance Evaluation of Deenbandhu Biogas Plants Installed in Various Regions of Punjab, India. Int.J.Curr.Microbiol.App.Sci. 9(02): 185-193. doi: https://doi.org/10.20546/ijcmas.2020.902.023 